\title{
Geology and Exploration History of Kimberlites and Related Rocks in South Australia
}

\author{
Steven A. Cooper ${ }^{1,2}$ \& Brian J. Morris ${ }^{3}$ \\ ${ }^{1}$ GEMOC ARC National Key Centre, Macquarie University, NSW, 2109, Australia \\ ${ }^{2}$ Orogenic Exploration Pty Ltd, Burwood, Vic, 3125, Australia \\ ${ }^{3}$ South Australia Geological Survey, PIRSA, Adelaide, SA, 5000, Australia
}

There are currently around 200 kimberlitic occurrences known in South Australia. The kimberlites occur in seven discrete provinces spanning a distance of 400 kilometres across South Australia (Figure 1). By the early 1980s all the known outcropping kimberlitic intrusions had been located while subsequent discoveries have generally been concealed bodies located by aeromagnetic surveys.

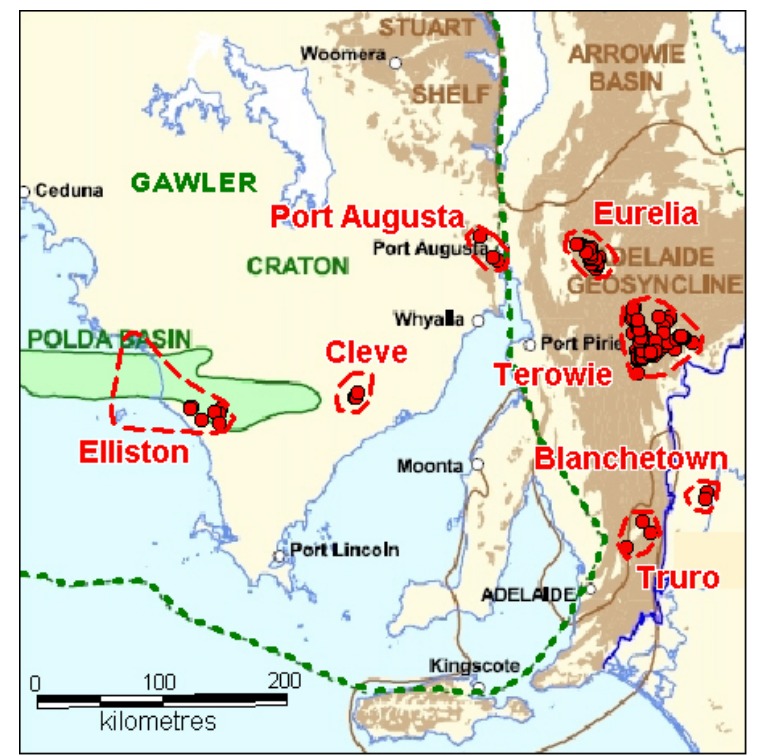

Figure 1. Kimberlitic Provinces in South Australia

\section{ELLISTON PROVINCE}

On the western Eyre Peninsula are the nine Mt Hope kimberlites. All were discovered in 1990-1991 by drilling discrete aerial magnetic targets after regional surface loam heavy mineral samples confirmed the presence of indicator grains in the area. Due to complex cover of Upper Jurassic fluvial clayey sandstones and lignite, Tertiary poorly sorted fluvial sands and inter-bedded clays, and extensive Quaternary calcarentites and calcrete, surface indicator patterns are displaced and dispersed away from the kimberlites. The kimberlites are hypabyssal and diatreme facies, porphyritic, possible monticellite?-phlogopite kimberlites. Microprobe studies of chromites from some Mt Hope bodies show strong evidence of mantle metasomatism.
This Province also includes the North Venus Bay (north side Polda Basin) and Flinders Island kimberlite indicator anomalies. Flinders Island is unique in having large numbers of all types of indicator minerals, including diamonds within the island soil (Cooper, 2002). Source rocks have not yet been located in these two areas.

\section{CLEVE}

The eastern Eyre Peninsula contains the three Cleve kimberlite dykes (Wyatt et al., 1991), first drilled in 1986. Stream and loam grid sampling narrowed down the location, with final costean and drilling based on ground magnetics and surface indicator counts. Groundmass perovskite U-Pb was used to obtain an 180 \pm 3 Ma date on the Cleve 01 kimberlite (Bristow, unpublished data, in Wyatt et al., 1991).

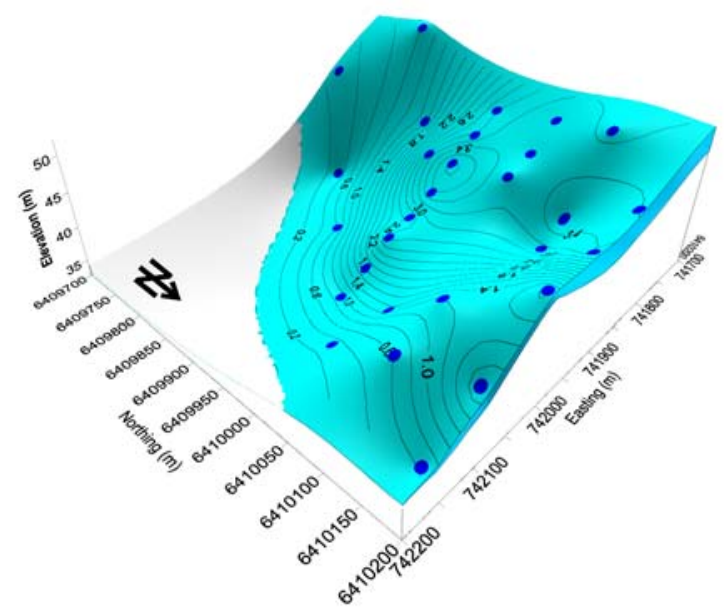

Figure 2. Model of the Sugarloaf Dam kimberlite sill based on drillhole intersections (dark blue circles), with thickness contours overlain. Image is looking southwest.

\section{PORT AUGUSTA}

The kimberlites in this area comprise two sills, the El Alamein, and Sugarloaf Dam sills. Recent work has shown the previously separated El Alamein East and West sills are actually the same body and appear to be nearly horizontal. The Sugarloaf Dam sill thickens towards the northwest and is a gentle syncline with fold axis plunging shallowly towards the southeast (Figure 2). Both sills were discovered in 1973 using surface loam sampling at ever-increasing density. 


\section{EURELIA PROVINCE}

The province comprises a number of kimberlite dykes, with the first 12 discovered described by Scott Smith et al. (1984). They are centred around the town of Eurelia, north of Orroroo, in the Adelaide Geosyncline. The diamondiferous Eurelia K07 kimberlite dyke and blow has been the most extensively studied of this province. The petrological descriptions by Scott Smith et al. (1984) came from the vertical drillhole DHR3 completed by Stockdale Prospecting during 1981.

The first kimberlitic rock dated in South Australia was from drillhole CD010, northwest from Eurelia (sample 75210434 in Stracke et al., 1979). Further dykes, some diamondiferous, are still being found currently in this area based on detailed high-resolution magnetic surveys. The Eurelia Province also includes dykes of carbonatitic affinity exposed in creek banks in the Walloway Diapir area discovered by Tucker \& Collerson (1972).

\section{TEROWIE PROVINCE}

Most of the kimberlites were located by De Beers during the period 1969-1972. This Province contains some of the first true kimberlite rocks found in the State, and probably in Australia. Exploration primarily involved the collection of alluvial heavy mineral samples. The Calcutteroo, Mittopitta, Pine Creek and other kimberlites were discovered by heavy mineral sampling, mapping along gullies, then shallow drilling. A large number of recent discoveries has been made by using high-resolution magnetic surveys. Some of these have been confirmed as weakly diamondiferous in the northern part of the province. It is possible the province merges with the Eurelia Province to the north, but currently there is still a $40 \mathrm{~km}$ gap between the two.

\section{TRURO}

Several small pipes are known from this area. They have been discovered by a combination of stream heavy mineral sampling and detailed magnetics.

\section{BLANCHETOWN}

By drilling blind magnetic targets in 1982, Rio Tinto discovered two kimberlitic intrusions below about 214 metres of Tertiary Murray Basin sediments.

As part of a project to systematically map and describe all the kimberlites and related rocks in South Australia, heavy mineral samples have been collected from a number of kimberlites in every kimberlitic field within each province. Not all are true kimberlites (see Cooper et al., 2007), and emplacement ages are not continuous across the State. Table 1 provides the first accurate location details for about half the State's kimberlites, some previously published, and many that have never been published.
The rate of discoveries of outcropping kimberlite has effectively dropped to zero in South Australia. Recent discoveries have all been under sediment cover. This trend will continue with the new frontier being the pervasively sediment-covered Gawler Craton. The discovery of diamonds and kimberlite indictor minerals, showing good mantle conditions on Flinders Island, suggests other parts of the Gawler Craton are prospective for diamonds, and not all areas have suffered the same metasomatism as the Mt Hope kimberlites (see Figure 3).

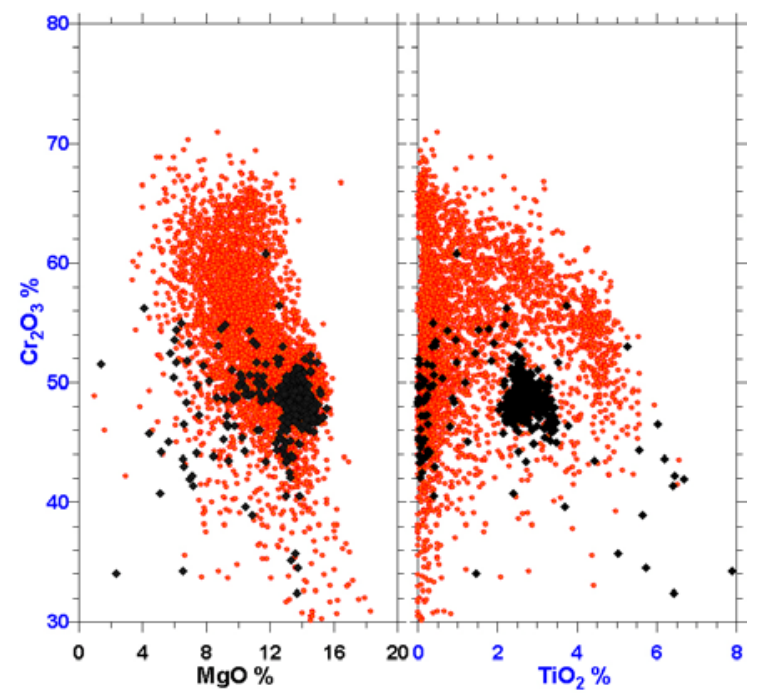

Figure 3. Microprobe analyses of chromite cores from Flinders Island (red, $\mathrm{n}=4559$ ) and Mt Hope kimberlites (black, $\mathrm{n}=418$ ) showing stark differences in population chemistry.

\section{References}

Cooper, S.A., 2002. New diamond field discovered in SA. MESA Journal 24, 6-9.

Cooper, S.A., Barron, B.J., \& Morris, B.J., 2007. Petrology of selected Kimberlite and Related Rocks in South Australia - Volume 1. South Australian Department of Primary Industries \& Resources, Report Book 2007/3.

Scott Smith, B.H., Danchin, R.V., Harris, J.W., \& Stracke, K.J. 1984. Kimberlites near Orroroo, South Australia. In: J. Kornprobst (ed.), Kimberlites and related rocks. Proceedings of the 3IKC, Vol. 1, Elsevier's Development in Petrology series, no. 11A, p. 121-142.

Stracke, K.J., Ferguson, J., \& Black, L.P., 1979. Structural Setting of Kimberlites in South-Eastern Australia. In Boyd, F.R. \& Meyer H.O.A., eds., Kimberlites, Diatremes and Diamonds, A.U.G., Washington, 71-91.

Tucker, D.H., \& Collerson, K.D., 1972. Lamprophyric intrusions of probable carbonatitic affinity from South Australia. Journal of the Geological Society of Australia 19, 387-391

Wyatt, B.A., Shee, S.R., Griffin, W.L., Zweistra, P., \& Robison, H.R., 1994. The petrology of the Cleve kimberlite, Eyre peninsula, South Australia. In: Meyer, H.O. \& Leonardos, O.H. (eds) Kimberlites, Related Rocks and Mantle Xenoliths. CPRM Special Publication $1 \mathrm{~A}, 62-79$. 
Table 1. Kimberlite locations in South Australia. Coordinates are in WSG84 Datum.

\begin{tabular}{|c|c|c|c|c|c|c|c|}
\hline Name & Longitude & Latitude & Province & Name & Longitude & Latitude & Province \\
\hline 6633RS99 & 138.502979 & -32.482167 & Eurelia & Mulga Creek 01 & 139.0165919 & -33.1884164 & Terowie \\
\hline Angaston 01 & 139.1241498 & -34.5127939 & Truro & Mungibbie 01 & 138.964993 & -33.2764351 & Terowie \\
\hline Calcuteroo 01 & 139.1998847 & -33.1198229 & Terowie & Mungibbie 02 & 138.96248 & -33.26937 & Terowie \\
\hline Calcuteroo 02 East & 139.1939805 & -33.1312899 & Terowie & Mungibbie 03 & 138.960037 & -33.263402 & Terowie \\
\hline Calcuteroo 02 West & 139.195045 & -33.131248 & Terowie & Mungibbie 04 & 138.961552 & -33.2542966 & Terowie \\
\hline Calcuteroo 02a & 139.19509 & -33.1306 & Terowie & Mungibbie 05 & 138.9615524 & -33.2534792 & Terowie \\
\hline Calcuteroo 02b & 139.1946464 & -33.1309962 & Terowie & Pandappa 02a & 139.1913702 & -33.1528923 & Terowie \\
\hline Calcuteroo 03 & 139.1934772 & -33.1338799 & Terowie & Pandappa 02b & 139.191531 & -33.1528946 & Terowie \\
\hline Calcuteroo 04a & 139.19526 & -33.1090021 & Terowie & Pandappa 03 & 139.1858215 & -33.1615052 & Terowie \\
\hline Calcuteroo 04b & 139.1955487 & -33.1095565 & Terowie & Pandappa 04 & 139.2022608 & -33.1440677 & Terowie \\
\hline Calcuteroo 04c & 139.1955385 & -33.1090062 & Terowie & Pine Creek 01 & 139.272983 & -33.187521 & Terowie \\
\hline Calcuteroo 04d & 139.1972185 & -33.111222 & Terowie & Pine Creek 02 & 139.26841 & -33.164186 & Terowie \\
\hline Calcuteroo 04e & 139.201655 & -33.1112229 & Terowie & Pine Creek 03 & 139.278645 & -33.1653394 & Terowie \\
\hline Calcuteroo 04f & 139.1986065 & -33.1109445 & Terowie & Pine Creek 04 & 139.289029 & -33.1678163 & Terowie \\
\hline Calcuteroo 05 & 139.2024833 & -33.1311424 & Terowie & Pine Creek 05 & 139.282834 & -33.1780949 & Terowie \\
\hline Calcuteroo 06 & 139.200228 & -33.130823 & Terowie & Pine Creek 07 & 139.280509 & -33.1691698 & Terowie \\
\hline Calcuteroo 09 & 139.19983 & -33.1203142 & Terowie & Pine Creek 08 & 139.267057 & -33.1916666 & Terowie \\
\hline CD10 & 138.4932691 & -32.5131946 & Eurelia & Pitcairn E1430 & 139.215642 & -32.967166 & Terowie \\
\hline Cleve 01 & 136.5228415 & -33.5945364 & Cleve & Pitcairn E1522 & 139.223599 & -32.961528 & Terowie \\
\hline Cleve 02 & 136.526441 & -33.58682 & Cleve & Pitcairn E1524 & 139.221259 & -32.963617 & Terowie \\
\hline Cleve 03 & 136.53833 & -33.558263 & Cleve & Pitcairn E1525 & 139.220408 & -32.966004 & Terowie \\
\hline El Alamein East & 137.733724 & -32.5838873 & Port Augusta & Pitcairn E1526 & 139.226606 & -32.962586 & Terowie \\
\hline El Alamein West & 137.685583 & -32.5666713 & Port Augusta & Pitcairn JS & 139.2226891 & -32.9626686 & Terowie \\
\hline Eurelia K01 & 138.543858 & -32.529034 & Eurelia & Sugarloaf Dam & 137.57183 & -32.421003 & Port Augusta \\
\hline Eurelia K02 & 138.530165 & -32.52092 & Eurelia & Terowie 02 & 138.911319 & -33.1520261 & Terowie \\
\hline Eurelia K03 & 138.577127 & -32.558836 & Eurelia & Terowie 03 & 138.911859 & -33.1514399 & Terowie \\
\hline Eurelia K04 & 138.5367888 & -32.5053248 & Eurelia & Terowie 04 & 138.908636 & -33.1693284 & Terowie \\
\hline Eurelia K05 & 138.609503 & -32.551722 & Eurelia & Terowie South 01 & 138.9111615 & -33.1769374 & Terowie \\
\hline Eurelia K06 & 138.600452 & -32.54298 & Eurelia & Terowie South 02 & 138.9122971 & -33.176 & Terowie \\
\hline Eurelia K07 & 138.51812 & -32.4907568 & Eurelia & Ulooloo 01 & 138.971863 & -33.364475 & Terowie \\
\hline Eurelia K08 & 138.500561 & -32.495936 & Eurelia & Ulooloo 02 & 138.889593 & -33.330424 & Terowie \\
\hline Eurelia K09a & 138.457337 & -32.47007 & Eurelia & Ulooloo 03 & 138.97229 & -33.3631978 & Terowie \\
\hline Eurelia K09b & 138.43612 & -32.456405 & Eurelia & Walloway X & 138.578986 & -32.628828 & Eurelia \\
\hline Eurelia K10 & 138.510325 & -32.490697 & Eurelia & Waupunyah 01 & 139.008044 & -33.2067808 & Terowie \\
\hline Eurelia K11 & 138.525414 & -32.479652 & Eurelia & Waupunyah 02 & 139.007763 & -33.2059468 & Terowie \\
\hline Eurelia K12 & 138.509485 & -32.483817 & Eurelia & Waupunyah 03 & 138.998696 & -33.208416 & Terowie \\
\hline Eurelia K13 & 138.502664 & -32.477538 & Eurelia & Waupunyah 04 & 138.994979 & -33.2081581 & Terowie \\
\hline FS03 & 139.5985849 & -34.2132765 & Blanchetown & Waupunyah 05 & 138.99257 & -33.2093838 & Terowie \\
\hline FS66 & 139.5820328 & -34.261727 & Blanchetown & Waupunyah 06 & 138.990135 & -33.2091276 & Terowie \\
\hline Hiles Lagoon 02 & 138.962136 & -33.200228 & Terowie & Waupunyah 07 & 139.0005443 & -33.1978952 & Terowie \\
\hline HLD1 262m & 138.964211 & -33.1902667 & Terowie & Waupunyah 08 & 139.001294 & -33.2 & Terowie \\
\hline HLD1 294m & 138.9640096 & -33.1901643 & Terowie & Waupunyah 09 & 138.998655 & -33.2017154 & Terowie \\
\hline Mittopitta 02 & 139.006858 & -33.254982 & Terowie & Waupunyah 10 & 138.999466 & -33.2089789 & Terowie \\
\hline Mittopitta 05 & 139.008736 & -33.248479 & Terowie & Waupunyah 17 & 139.0083145 & -33.2062261 & Terowie \\
\hline Mittopitta 06 & 139.0061373 & -33.2568661 & Terowie & Whyte Yarcowie 01 & 138.9632097 & -33.2343256 & Terowie \\
\hline Mittopitta Diatreme & 139.006752 & -33.252567 & Terowie & Whyte Yarcowie 02 & 138.9631125 & -33.2344358 & Terowie \\
\hline Mt Hope 01 & 135.1938877 & -33.7554577 & Elliston & Wonna 01 & 138.995836 & -33.2542279 & Terowie \\
\hline Mt Hope 01 Satellite & 135.1955375 & -33.7522312 & Elliston & Wonna 02 & 138.9960879 & -33.2493875 & Terowie \\
\hline Mt Hope 02 & 135.343791 & -33.785207 & Elliston & Wonna 03 & 138.996658 & -33.2483686 & Terowie \\
\hline Mt Hope 03 & 135.3563276 & -33.6824151 & Elliston & Wonna 04 & 138.996026 & -33.2483404 & Terowie \\
\hline Mt Hope 04 & 135.345096 & -33.709222 & Elliston & Wonna 05 & 138.993999 & -33.24465 & Terowie \\
\hline Mt Hope 05 & 135.300734 & -33.697874 & Elliston & Wonna 06 & 138.994109 & -33.2415913 & Terowie \\
\hline Mt Hope 06 & 135.10887 & -33.665019 & Elliston & Wonna 07 & 138.993553 & -33.2466413 & Terowie \\
\hline Mt Hope 07 & 135.091452 & -33.666395 & Elliston & Wonna 08 & 138.989136 & -33.2463278 & Terowie \\
\hline Mt Hope 08 & 135.097106 & -33.677629 & Elliston & Wonna 09 & 138.995366 & -33.2485562 & Terowie \\
\hline Mt Scrub 03 & 138.9366674 & -33.2970837 & Terowie & Wonna 18 & 139.0027011 & -33.2663608 & Terowie \\
\hline
\end{tabular}

